$$
\sum^{\prime} f^{(1)}\left(n_{1}\right) f^{(2)}\left(n_{2}\right) \cdots f^{(\nu)}\left(n_{\nu}\right)=(1+o(1)) D n^{\nu-1},
$$

also

$$
\sum_{m=1}^{n} f^{(1)}\left(m+k_{1}\right) f^{(2)}\left(m+k_{2}\right) \cdots f^{(v)}\left(m+k_{\nu}\right)=(1+o(1)) E n,
$$

$D$ and $E$ are given by a complicated expression.

STANFORD UNIVERSITY

\title{
ON A CLASS OF TAYLOR SERIES
}

\section{F. COWLING}

1. Introduction. Consider the Taylor series $\sum_{n=0}^{\infty} a_{n} z^{n}$. Suppose that the singularities of the function defined by the series all lie in certain regions of the complex plane and that the coefficients possess certain arithmetical properties. Mandelbrojt ${ }^{1}$ has shown that under restrictions of this nature it is possible to predict the form of the function defined by the series. This note is concerned with the establishing of a new method to obtain more general results of this nature.

2. The method. The method that is employed here is an adaptation of a method used by Lindelöf [2] in the problem of representation of a function defined by a series.

Let $f(z)$ be regular in a region $D$ of the complex plane. Suppose that there exists a linear transformation $t=h(z)$ which maps the region of regularity into a region which includes the unit circle of the $t$-plane in its interior. Let $z=g(t)$ be the inverse of this transformation. Then $F(t)=f(g(t))$ is regular in this region in the $t$-plane. For this note it is convenient to suppose that $z=0$ corresponds to $t=0$ in the mapping. We may expand $g(t)$ in a Taylor series about $t=0$ and obtain

$$
z=b_{1} t+b_{2} t^{2}+\cdots
$$

convergent for $t$ in absolute value sufficiently small. Let

$$
f(z)=\sum_{n=0}^{\infty} a_{n} z^{n}
$$

Received by the editors August 2, 1946.

${ }^{1}$ See, Mandelbrojt [3]. Numbers in brackets refer to the bibliography at the end of the paper. 
be the element of $f(z)$ at the origin. For $t$ in absolute value sufficiently small we may substitute (2.1) in (2.2) and obtain

$$
F(t)=f(g(t))=\sum_{n=0}^{\infty} C_{n} t^{n} .
$$

We have seen, however, that $F(t)=f(g(t))$ is regular in a region in the $t$-plane which includes the unit circle in its interior. Hence the radius of convergence of (2.3) is greater than one. Therefore we may write

$$
\limsup _{n \rightarrow \infty}\left(\left|C_{n}\right|\right)^{1 / n}<1
$$

As the $C_{n}$ are polynomial combinations of the $a_{n}$ and $b_{n}$ we see that under certain circumstances (2.4) may imply $C_{n}=0$ for $n$ greater than some $n_{0}$. For example, if the $C_{n}$ are all integers (2.4) implies the existence of an $n_{0}$ such that $C_{n}=0$ for $n>n_{0}$. It is also clear that if $C_{n}=C_{n}^{\prime}+i C_{n}^{\prime \prime}$ where $C_{n}^{\prime}$ and $C_{n}^{\prime \prime}$ are integers that the conclusion $C_{n}=0, n>n_{0}$, still holds. Under these circumstances we obtain upon substituting $t=h(z)$ in (2.3)

$$
f(z)=\sum_{n=0}^{n_{0}} C_{n}[h(z)]^{n} .
$$

3. Applications. We now proceed to the proof of the principal theorem.

THEOREM 3.1. If the series $f(z)=\sum_{n=0}^{\infty} a_{n} z^{n}$ has rational coefficients such that there exists an integer $L$ for which the quantities

$$
a_{0}, a_{1} L, a_{2} L^{2}, \cdots, a_{n} L^{n}, \cdots
$$

are integers, and if the function defined by the series is regular exterior to and on the circumference of a circle with center $\left(L K^{\prime} /\left(K^{\prime 2}+K^{\prime \prime 2}-1\right)\right.$, $\left.L K^{\prime \prime} /\left(K^{\prime 2}+K^{\prime \prime 2}-1\right)\right)$ and radius $L /\left(K^{\prime 2}+K^{\prime \prime 2}-1\right)$ where $K^{\prime}$ and $K^{\prime \prime}$ are integers, $K^{\prime 2}+K^{\prime \prime 2} \neq 1$, then the function defined by the series is of the form

$$
\frac{P(z)}{\left(L /\left(K^{\prime}+i K^{\prime \prime}\right)-z\right)^{n_{0}}}
$$

where $P(z)$ is a polynomial and $n_{0}$ is a positive integer.

It is easily shown that the transformation

$$
t=\frac{z}{L-\left(K^{\prime}+i K^{\prime \prime}\right) z}
$$


maps the region of regularity into a region containing the unit circle of the $t$-plane in its interior. By substituting the solution of (3.1) for $z$ in (2.2) and setting $K=K^{\prime}+i K^{\prime \prime}$ we have

$$
\begin{aligned}
F(t)=f(g(t)) & =\sum_{n=0}^{\infty} a_{n} \frac{L^{n} t^{n}}{(1+K t)^{n}} \\
& =\sum_{n=0}^{\infty} a_{n} L^{n} t^{n} \sum_{m=0}^{\infty} C_{-n, m}(K t)^{m} .
\end{aligned}
$$

Then since the series are absolutely convergent, we obtain

$$
\begin{aligned}
F(t)=\sum_{n=0}^{\infty}\left[a_{n} L^{n} C_{-n, 0}+C_{-n, 1} K L^{n-1} a_{n-1}\right. & +\cdots \\
& \left.+a_{0} C_{-n, n} K^{n}\right] t^{n},
\end{aligned}
$$

(3.2) $F(t)=\sum_{n=0}^{\infty} C_{n} t^{n}$

Here $C_{n}=C_{n}^{\prime}+i C_{n}^{\prime \prime}$ where $C_{n}^{\prime}$ and $C_{n}^{\prime \prime}$ are integers, for the $C_{-n, m}$ are binomial coefficients and $a_{n} L^{n}$ is an integer for all $n \geqq 0$ by hypothesis. Also $K^{\prime}$ and $K^{\prime \prime}$ are integers. Hence, from the discussion in $\$ 2$ it follows that

$$
\limsup _{n \rightarrow \infty}\left|C_{n}\right|^{1 / n}<1
$$

implies the existence of a number $n_{0}$ such that $C_{n}=0$ for $n>n_{0}$. Therefore upon substituting (3.1) in (3.2) we have

$$
f(z)=\sum_{n=0}^{n_{0}} C_{n}\left(\frac{z}{L-K z}\right)^{n}=\frac{P(z)}{\left(L /\left(K^{\prime}+i K^{\prime \prime}\right)-z\right)^{n_{0}}} .
$$

This completes the proof of the theorem. If now we choose $K^{\prime \prime}=0$ and $K^{\prime}=L$ we have the theorem of Mandelbrojt [3]. This proof of Mandelbrojt's theorem has some points in common with a proof of the same theorem due to Achyser [1]. ${ }^{2}$ If in addition the $a_{n}$ are all integers we may set $L=1$ and have a new theorem.

We now proceed to the proof of the following theorem.

THEOREM 4.1. If the series $\sum_{n=0}^{\infty} a_{n} z^{n}$ has rational coefficients such that there exists an integer $L$ for which the quantities

$$
a_{0}, a_{1} L, a_{2} L^{2}, \cdots, a_{n} L^{n}, \cdots
$$

2 The author is indebted to the referee for this observation. 
are integers and if the function defined by the series is regular in the halfplane $R(z) \leqq L / 2$ including the point at infinity then the series defines a function of the form

$$
\frac{P(z)}{(L-z)^{n_{0}}}
$$

where $P(z)$ is a polynomial and $n_{0}$ is a positive integer.

From the hypothesis it is easily seen that the transformation

$$
t=\frac{z}{L-z}
$$

maps the region of regularity into a region which includes the unit circle in the $t$-plane in its interior. Upon solving (3.3) for $z$ and substituting in (2.2) we obtain

$$
\begin{aligned}
F(t) & =f\left(\frac{L t}{1+t}\right)=\sum_{n=0}^{\infty} a_{n}\left(\frac{L t}{1+t}\right)^{n} \\
& =\sum_{n=0}^{\infty} a_{n} t^{n} L^{n} \sum_{m=0}^{\infty} C_{-n, m}(t)^{m}=\sum_{n=0}^{\infty} C_{n} t^{n} .
\end{aligned}
$$

Then by the same arguments employed in Theorem (3.1) it follows that there exists an $n_{0}$ such that $C_{n}=0$ for $n>n_{0}$. Therefore by substituting from (3.3) in (3.4) we have

$$
f(z)=\sum_{n=0}^{n_{0}} C_{n}\left(\frac{z}{L-z}\right)^{n}=\frac{P(z)}{(L-z)^{n_{0}}},
$$

where $P(z)$ is a polynomial and $n_{0}$ is a positive integer. This completes the proof of the theorem.

\section{BIBLIOGRAPHY}

1. Achyeser, Über eine Anwendung der Eulerschen Transformation, Bulletin de l'Académie des Sciences de l'Oucraine vol. 1 (1925) p. 32.

2. E. Lindelöf, Remarques sur un principe general de la theorie des fonctions analytiques, Acta Societatis Fennicae, vol. 24, no. 7, Helsingfors, 1898.

3. S. Mandelbrojt, Modern researches on the singularities of functions defined by Taylor's series, The Rice Institute Pamphlet, vol. 14, no. 14, 1927.

LEHIGH UNIVERSITY 